\title{
Efeito acaricida in vitro do extrato hidroalcoólico de Himatanthus sucuuba contra Rhipicephalus microplus
}

(In vitro acaricidal effect of Himatanthus sucuuba hydroalcoholic extracts against Rhipicephalus microplus)

Lew Kan Sprenger, Marcela Sigolo Vanhoni, Elane Guerreiro Giese, Jeannie Nascimento dos Santos, Marcelo Beltrão Molento ${ }^{1}$

${ }^{1}$ Correspondência: molento@ufpr.br

RESUMO: O objetivo deste estudo foi avaliar a eficácia in vitro de extratos hidroalcóolicos de Himatanthus sucuuba frente a larvas e fêmeas ingurgitadas do carrapato Rhipicephalus microplus. Os fitoterápicos foram produzidos com percolação de látex, caule e folhas, sendo posteriormente liofilizados. Foi realizado o teste do pacote de larvas, TPL em concentrações crescentes $\left(0,4-204,8 \mathrm{mg} \cdot \mathrm{mL}^{-1}\right)$ e o teste de imersão de adulto, TIA (6,2-396,8 mg. $\left.\mathrm{mL}^{-1}\right)$ ambos com seis repetições. Foi realizada a análise fitoquímica completa dos três extratos. No TPL e TIA, o fitoterápico produzido com látex demonstrou os melhores resultados, com DL50 de 21,4 e 105,2 mg. $\mathrm{mL}^{-1}$, respectivamente. Os dados da marcha fitoquímica, aliado aos resultados dose-dependente obtidos nos testes in vitro evidenciam que o extrato produzido a partir do látex possui potencial para combater o $R$. microplus. Novos estudos devem ser realizados buscando maximizar a eficácia deste extrato, uma vez que foram encontrados resultados promissores.

Palavras-chave: Fitoterapia, janaguba, janajuba, carrapato, resistência parasitária

ABSTRACT: The aim of this study was to evaluate the in vitro efficacy of the Himatanthus sucuuba hydroalcoholic extracts against larvae and engorged female of Rhipicephalus microplus. The extracts were prepared by percolation from latex, stems and leaves in absolute alcohol and lyophilized. We performed the larval packet test, LPT in serial concentrations $\left(0.4-204.8 \mathrm{mg} \mathrm{mL}^{-1}\right)$ and adult immersion test, AIT (6.4-396.8 mg. $\mathrm{mL}^{-1}$ ) using six replicates. The complete phytochemical composition was determined to all the extracts. From the LPT and TIA, the latex demonstrated the best results, with an LD 50 of 21.4 and $105.2 \mathrm{mg} \cdot \mathrm{mL}^{-1}$, respectively. The phytochemical data, combined with the dose-dependent results obtained from the in vitro tests demonstrates that this extract has potential to control $R$. microplus. Further studies should be conducted to maximize the effectiveness of the latex portion of the plant.

Key Words: Janaguba, janajuba, parasitic resistance, phytotherapy 


\section{INTRODUÇÃO}

A bovinocultura brasileira é composta hoje por mais de 209 milhões de animais (IBGE, 2015), contudo a produtividade do setor é conhecidamente afetada por graves entraves sanitários. Entre os principais agravantes está 0 parasitismo por Rhipicephalus microplus, carrapato presente em diversas regiões brasileiras. Este ectoparasita, causa prejuízos econômicos anuais de aproximadamente US $\$ 3,24$ bilhões para a pecuária nacional, sendo decorrentes principalmente da queda na produção de leite e carne, danos no couro por reações inflamatórias nos locais de fixação do carrapato, e pela transmissão de agentes infecciosos, principalmente Anaplasma e Babesia. (Grisi et al., 2014).

No Brasil, o uso de acaricidas é realizado de maneira supressiva, sem considerar a epidemiologia e o ciclo do parasito. Neste cenário, a aceleração do processo de seleção para resistência do carrapato aos fármacos é motivo de grande preocupação. Além disto, o uso de muitos dos carrapaticidas atuais afetam o meio ambiente e outros organismos, fazendo com que se busque alternativas para os produtos químicos comerciais, que visem minimizar os impactos econômicos e ambientais do uso de pesticidas sintéticos (Molento et al., 2013; ABBAS et al., 2014).

Nesse contexto, diversas tecnologias estão sendo desenvolvidas, e dentre elas a fitoterapia pode ser considerada uma estratégia promissora. Dentre as diversas plantas pesquisadas na região amazônica, destaca-se a Himatanthus sucuuba. Pertencente à família Apocynaceae, é uma árvore de grande porte, nativa da região Amazônica e conhecida popularmente como Sucuuba, Janaguba ou Janajuba. O látex e o chá da casca da planta são utilizados pelos povos da Amazônia contra tumores, úlceras, asma e tuberculose. Diversos estudos farmacológicos foram realizados com esta planta, sendo indicada para o tratamento de feridas em geral (Villegas et al., 1997), tumores inespecíficos (Wood et al., 2001), resfriados (Souza et al., 2006), anemia, tratamento de gastrites, leishmaniose (Castillo et al., 2007), artrite, inflamações (Silva et al., 1998a; Miranda et al., 2000) e no combate a fungos (Silva et al., 1998b). Assim, o presente estudo teve o objetivo de determinar os efeitos de compostos da $H$. sucuuba sob a ovipostura de teleóginas de $R$. microplus através da utilização de extratos obtidos de sua folha, caule e látex. 


\section{MATERIAL E MÉTODOS}

A presente pesquisa foi conduzida no laboratório de microbiologia da Universidade do Contestado, Campus Canoinhas, durante o mês de junho, 2015. Amostras de caule, folhas e látex de $H$. sucuuba foram obtidas no Mercado Ver-o-Peso, Belém, Pará. As amostras biológicas estão depositadas no herbário da Universidade Federal do Paraná sob o número 543-2. Para a extração hidroalcoólica utilizou-se um frasco de cor âmbar, vedado em toda a extensão lateral com papel alumínio, no qual foram colocados, separadamente, $80 \mathrm{~g}$ de cada porção vegetal (folha, caule e látex) isoladamente, com $800 \mathrm{~mL}$ de álcool etílico $70 \%(\mathrm{v} / \mathrm{v})$. Na sequência, o conteúdo ficou trinta dias retido em refrigerador a $4^{\circ} \mathrm{C}$, sendo realizada a agitação recorrente do frasco. Concluída esta etapa, com auxílio de uma bomba de vácuo, realizou-se a filtração em funil com papel filtro para um frasco tipo Kitasato. Posteriormente a obtenção dos extratos, estes foram concentrados em evaporador rotatório, sob pressão reduzida à uma temperatura inferior a $30^{\circ} \mathrm{C}$, sendo liofilizados em seguida. Os rendimentos em relação ao material vegetal foram de: 5,$14 ; 4,12$ e $2,15 \%$, para folha, caule e látex, respectivamente.
Os extratos fitoterápicos foram dissolvidos em água destilada na concentração de $10 \mathrm{mg} / \mathrm{mL}$, para a realização da determinação qualitativa dos seguintes metabólitos, de acordo com a metodologia de Matos (1997): alcalóides, antocianinas, antocianidinas, catequinas, esteróides, fenóis, flavonóides, leucoantocianidinas, resinas, saponinas, taninos e triterpenos.

A atividade sequestrante do radical DPPH foi determinada segundo a metodologia de Blois (1958), com modificações. Em microtubos de Eppendorf, foram depositados $300 \mu \mathrm{l}$ dos extratos em diferentes concentrações (10-640 $\mathrm{\mu g} / \mathrm{mL}$ ) junto a $100 \mu \mathrm{l}$ de solução etanólica de DPPH $(0,1 \mathrm{mM})$. Para o branco da amostra foi acrescentado $100 \mu \mathrm{l}$ de etanol (EtOH) ao invés do DPPH, enquanto no branco do método foi utilizado $300 \mu \mathrm{EtOH}$ no lugar das amostras. Os tubos foram agitados em vórtex e em seguida se transferiu $200 \mu \mathrm{l}$ do preparado para uma placa de 96 poços. O preparado foi incubado no escuro, em temperatura ambiente pelo tempo de $30 \mathrm{~min}$. Transcorrido o tempo, a absorbância de cada solução foi mensurada no espectrofotômetro a $515 \mathrm{~nm}$. A atividade antioxidante das amostras foi expressa como Cl50 (concentração inibitória), que 
foi determinada como a concentração, em $\mu \mathrm{g} / \mathrm{mL}$, de amostra necessária para inibir a formação de radicais DPPH em $50 \%$. O ácido ascórbico foi utilizado como controle positivo.

A atividade citotóxica dos extratos foi avaliada seguindo a metodologia adaptada de Meyer et al. (1982), onde ovos de Artemia salina, um microcrustáceo, foram colocados em aquário, contendo $2 \mathrm{~L}$ de água filtrada $\mathrm{e}$ sal marinho (38g/L) sob aeração constante e temperatura controlada de $28^{\circ} \mathrm{C}$. Após $48 \mathrm{~h}$ de incubação, as larvas/metanáuplios eclodiram e foram transferidas ( $n=10 /$ grupo) para placas de 24 poços, contendo os extratos diluídos em água marinha (descrição acima) com DMSO ( $1 \% \quad \mathrm{v} / \mathrm{v})$, em concentrações variando de 31,75 a $8000 \mu \mathrm{g} / \mathrm{mL}$. O grupo controle negativo foi preparado somente com água marinha e DMSO ( $1 \% \mathrm{v} / \mathrm{v})$. No controle positivo foi utilizado sulfato de quinidina. Os testes foram produzidos em triplicata. Após 24h de contato, realizouse a contagem do número de larvas vivas. Aquelas que permaneceram imóveis após a agitação da placa, por um período superior a 15 segundos, foram consideradas mortas. Com base nesse valor, foi calculada a CL50. O critério de toxicidade dos extratos foi estabelecido de acordo com Déciga-
Campos et al. (2007), onde valores $>1000 \mu \mathrm{g} / \mathrm{mL}$ são considerados não tóxicos, de $\geq 500$ a $\leq 1000 \mu \mathrm{g} / \mathrm{mL}$, são fracamente tóxicos e $<500 \mu \mathrm{g} / \mathrm{mL}$ são considerados claramente tóxicos.

Fêmeas ingurgitadas de $R$. microplus medindo $\geq 4.6 \mathrm{~mm}$ foram coletadas de bovinos Holandês $x$ Jersey naturalmente infestados, em propriedades rurais do município de Canoinhas, SC. Os animais não tinham recebido acaricidas alopáticos por no mínimo cinco meses. As fêmeas foram lavadas com água e secas em papel toalha, para então serem selecionadas morfologicamente e utilizadas no teste de imersão de adulto, TIA ou mantidas em laboratório $\left(28^{\circ} \mathrm{C}\right.$ e umidade relativa $\geq 80$ ) durante 16 dias, até realizarem a ovipostura. Os respectivos ovos foram utilizados para executar o teste do pacote de larvas, TPL.

Para a realização do TIA, várias diluições $\left(6,2\right.$ - 396,8 mg. $\left.\mathrm{mL}^{-1}\right)$ dos extratos foram testadas, de acordo com Drummond et al. (1973). Fêmeas ingurgitadas foram pesadas e imersas durante 5 min na respectiva diluição. Três réplicas, cada uma contendo dez teleóginas, foram utilizados em cada diluição. Soluções de etanol $95 \%$ com DMSO $1 \%$ foram usadas como controle negativo e cipermetrina (5 mg. $\mathrm{mL}^{-1}$ ) como controle positivo. As fêmeas foram 
recuperadas, secadas utilizando papel toalha e colocadas em tubos plásticos $(25 \times 90 \mathrm{~mm})$ separadamente. Estes tubos foram incubados em estufa tipo BOD e mantidos a $28^{\circ} \mathrm{C}$ com umidade relativa $\geq 80 \%$. Após 20 dias, os parâmetros reprodutivos foram calculados.

O TPL foi conduzido de acordo com Leite et al. (1988). Cerca de 200 larvas foram colocadas em um envelope de papel filtro dobrado não impregnado $\left(8 \times 8 \mathrm{~cm}^{2}\right)$ e selados em um lado com fitas adesivas, formando um pacote triangular aberto. $O$ envelope foi colocado em uma incubadora tipo BOD e mantida $\left(28^{\circ} \mathrm{C}\right.$ com umidade relativa $\geq 80 \%$ ) durante $24 \mathrm{~h}$. Os testes foram realizados em concentrações que variaram de 0,4 - 204,8 mg. $L^{-1}$, utilizando soluções de etanol $99 \%$ com DMSO $1 \%$ como controle negativo e cipermetrina (5 mg. $\mathrm{mL}^{-1}$ ) como controle positivo. $O$ teste foi realizado em triplicata para todas as concentrações do acaricida. Os pacotes foram removidos após $24 \mathrm{~h}$, e as larvas vivas e mortas de cada pacote foram contabilizadas.

Para o TIA, o índice de produção de ovos (IPE) (Bennet, 1974), redução da oviposição (RO) (Roulston et al., 1968) e a eficácia do extrato (E\%) (Drummond et al., 1973) foram calculados usando as seguintes fórmulas:

IPE=(peso dos ovos/peso da fêmea ingurgitada) $\times 100$

RO=(IPE grupo controle-IPE grupo experimental/IPE grupo controle) $\times$ 100

$\mathrm{E}=$ (IER grupo controle-IER grupo experimental)/(IER grupo controle $\times 100$ )

Índice de eficiência reprodutiva (IER) $=$ (peso da massa de ovos $x$ porcentagem de eclodibilidade dos ovos / peso das fêmeas ingurgitadas) $\times 20.000$

Para ambos os testes, as concentrações letais $\left(\mathrm{CL}_{50}\right)$ foram calculadas usando o software GraphPad Prism 5 para Windows (San Diego, CA, EUA). As diferenças entre as médias foram comparadas pelo teste de Tukey $(P<0,05)$.

\section{RESULTADOS E DISCUSSÃO}

A Floresta Amazônica é a maior floresta tropical do planeta, possuindo uma flora que contem de cerca de $20 \%$ das espécies vegetais do planeta, contabilizando no Brasil cerca de 40 mil plantas (POSSIMOSER et al., 2013). Pesquisas voltadas para o estudo e a avaliação de novos medicamentos 
tendo como matéria prima vegetais da flora amazônica vêm crescendo exponencialmente. Estudos fitoquímicos e novas metodologias de pesquisas têm sido incorporadas a produção, visando o entendimento da ação antiparasitária pela presença de biocompostos nos extratos vegetais (CHAGAS, 2008).

Em ambos os extratos, foram detectadas a presença dos seguintes metabólitos: fenóis, flavonoides, taninos, esteróides, triterpenos, resinas, catequinas e alcalóides. Ao passo que antocianinas, antocianidinas, leucoantocianidinas e saponinas não foram encontrados em quantidade mensurável nos fitoterápicos produzidos. Estes dados corroboram com a literatura científica (Rodrigues et al., 2010; Luz et al., 2014), onde os autores realizaram a marcha fitoquímica qualitativa e quantitativa, está por cromatografia líquida de alta eficiência, e observaram os mesmos composto químicos anteriormente citados.

No ensaio antioxidante, foi observado que a $\mathrm{CE}_{50}$, dos fitoterápicos produzidos a base de folha, caule e látex, foram respectivamente: $40,16( \pm 1,88), 32,31$ $( \pm 4,54)$ e $29,44( \pm 3,57) \mathrm{mg} \cdot \mathrm{mL}^{-1}$, ao passo que o padrão demonstrou 0,68 $( \pm 0,14) \quad \mathrm{mg} \cdot \mathrm{mL}^{-1}$. Estes resultados evidenciam que os principais compostos presentes quantitativamente na planta são derivados de fenóis, sendo que o principal componente fenólico contido na $H$. suucuba são os flavonóides. Estudos demonstraram que além ser o maior responsável pela atividade antioxidante, os flavonóides possuem diversas propriedades biológicas, como a ação acaricida (Madzimure et al., 2011). These authors used Lippia javanica aqueous leaf extracts, a plant rich in flavonoids, and observed that efficacy of $L$. javanica treatments $(5 ; 10$ and $20 \% \mathrm{w} / \mathrm{v}$ ) against engorged ticks were not significantly different from those of amitraz $(0,2 \% \mathrm{w} / \mathrm{v})$.

O efeito acaricida dos taninos, composto presente no extrato de $H$. sucuuba produzido neste estudo, possui ação acaricida comprovada por FernandezSala et al. (2011). Estes autores testaram quarto plantas ricas em taninos, Acacia pennatula, Piscidia piscipula, Leucaena leucocephala e Lysiloma latisiliquum, demonstrando uma eficácia de 35; 39,21; 29 e 69,34\%, respectivamente. Estes dados também foram reforçados por Juliet et al. (2011), usando extrato aquoso de Jatropha curcas. Em nematodas, os taninos agem reduzindo a excreção de ovos e diminuindo a fertilidade das fêmeas adultas (Molan et al., 2003; Sprenger et al., 2015), sendo possível que um 
In vitro acaricidal effect of Himatanthus sucuuba hydroalcoholic extracts against Rhipicephalus microplus

mecanismo similar ocorra em significativa $(P<0,05)$ na oviposição e

ectoparasitos.

Cantrell et al. (2005) estudaram a ação inseticida de terpenoides presentes na Callicarpa japonica Thunb, e começaram a especular que estes compostos também poderiam possuir ação carrapaticida. Hoje sabe-se que os terpenóides estão envolvidas no mecanismo de defesa dos vegetais superiores contra ácaros herbívoros e outros artrópodes patogénicos (Garcia et al., 2012). Particularmente, este composto apresenta mecanismo de ação semelhante aos repelentes compostos por DEET (N-Dimetil-metatoluamida) (Jordan et al., 2012).

Os resultados do TPL encontram-se na Tabela 1, sendo que a DL50 dos extratos produzidos com látex, caule e folha foi, respectivamente, 21,$4 ; 183,8 ; 784,4$ $\mathrm{mg} \cdot \mathrm{mL}^{-1}$.

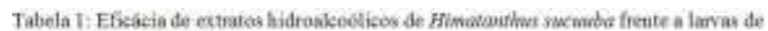
Rhipicephaslis micropitus.

\begin{tabular}{|c|c|c|c|}
\hline 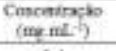 & Litex. & Conk & Fodtum \\
\hline Q.4 & $2,3 \pm 0,0$ & $20=1,22$ & $1,1 \pm \theta, 4$ \\
\hline 0,8 & $3,0 \pm 1,2$ & $2,4 \pm 1,40$ & $1,4=0,8^{\circ}$ \\
\hline 18 & $3,543,7$ & $30 a t \%$ & $2,141,1^{*}$ \\
\hline 3.2 & $14,200,9$ & $5,0+1, F^{*}$ & $4,0=07$ \\
\hline 0.4 & $41,5=2,9$ & $5,4 * 1,5^{2}$ & $7,8 * 2,14$ \\
\hline 128 & $4,3=1,7$ & $17,5+3,4$ & $17,224,11$ \\
\hline 25.6 & $59,-2,9$ & $32,943,7$ & $33,0=4,7 \cdots$ \\
\hline 512 & $\theta 9,4=5,4$ & $39.1+2,0$ & $-35,1 \pm 2,7$ \\
\hline $102+$ & $72,2=8,2^{2}$ & $44,1 \pm 2,35$ & at, $1 \pm 4,8$ \\
\hline 2048 & $p 8, t<1, \theta^{\circ}$ & $55,+427$ & $4,1 \pm 1,4$ \\
\hline 04.40 & 20.4 & 183,5 & $73+4$ \\
\hline c. & $0,0,0,0$ & D.0.00 & 0,0000 \\
\hline C+ & $98,40,8$ & $98, t+0, s^{t}$ & $92,+60 \%$ \\
\hline
\end{tabular}

Letras diferentes entre as lacunas indicam valones estatisticanente diferentes $(P-0.05$ )

Já no TIA (Tabela 2, 3 e 4) a melhor DL50 destes mesmos extratos foi do fitoterápico produzido com látex (105,2 mg. $\left.m L^{-1}\right)$. Houve uma redução eclodibilidade dos ovos das fêmeas ingurgitadas tratadas com o fitoterápico produzido com látex em concentrações acima de 396,8 mg.mL $\mathrm{m}^{-1}$. Um efeito carrapaticida dose-dependente foi observado para os três extratos utilizados na presente pesquisa.

\begin{tabular}{|c|c|c|c|c|}
\hline $\begin{array}{l}\text { Concertracto } \\
\text { (ang nuL - }\end{array}$ & $\begin{array}{l}\text { Inatire de jnotipso } \\
\text { de ovioe }\end{array}$ & 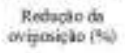 & 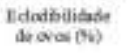 & If fisich \\
\hline 0.2 & $65,5 * 1,7$ & 6.4 & $90,1=1,9$ & $8.8+1,2$ \\
\hline 12,4 & $63,1+1,5$ & $9 n$ & $94,6=0,8$ & $13,502,7$ \\
\hline 24,8 & $62,2+24$ & 105 & $95,0=20$ & $161+13$ \\
\hline 49,6 & $S E, 0 \pm 1,7$ & 17,1 & $88,8=1,4$ & $25,3=21$ \\
\hline 90,2 & $46,9=1,7$ & 40.3 & $78,8=2,3$ & $4+341.1$ \\
\hline 108.4 & $22+121$ & 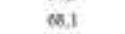 & $n s \in=3,3$ & $\pi, 4=23$ \\
\hline 2006 & $11,7 * 2,7$ & 893 & $50,7=1,7$ & $90,2-21$ \\
\hline c. & $70,200,8$ & $\because$ & $98,3=1,1$ & 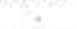 \\
\hline c. & $98,2+1,4$ & 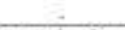 & $s 44=13$ & $95,8=1,1$ \\
\hline
\end{tabular}
lecunss indicath valores estatisticamente difarestes (P-0.05)

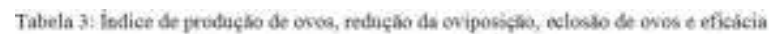

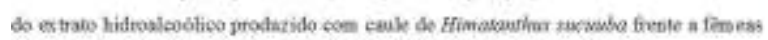

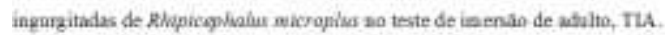

\begin{tabular}{|c|c|c|c|c|}
\hline $\begin{array}{c}\text { Concantracào } \\
\left(\text { menal }{ }^{-1}\right)\end{array}$ & $\begin{array}{l}\text { Indice de prodoplo } \\
\text { de ovos }\end{array}$ & $\begin{array}{l}\text { Reduçăo da } \\
\text { oviposiça (ta) }\end{array}$ & $\begin{array}{l}\text { Eclodibilisfade } \\
\text { de avos }(0)\end{array}$ & Efieacia \\
\hline 6.2 & $68,2+1,3$ & 27 & $98,1 \pm 1,1$ & $3,1 \pm 0,9$ \\
\hline 12.4 & $67,6 \pm 1,1$ & 3,3 & $97,6 \pm 1,4$ & $4, s \pm 1,3$ \\
\hline 24.8 & $65,2 \pm 1,4$ & 6.8 & $93,6 \pm 1,1$ & $11,6 \pm 2,1$ \\
\hline 49.6 & $65,0 \pm 1,9$ & 7,1 & $92,5 \pm 1,6$ & $12,8 \pm 1,5$ \\
\hline 99.2 & $57,9 \pm 1,2$ & 172 & $88,8 \pm 4,5$ & $25,1+2,0$ \\
\hline 198.4 & $\$ 7,3 \pm 0,7$ & 18.4 & $86,2+2,0$ & $28,4 \pm 1,3$ \\
\hline 395.8 & $53,6 \pm 1,9$ & 23,1 & $81,9 \pm 2,2$ & $33,7 \pm 2,1$ \\
\hline c- & $70,2+0,8$ & . & $98,8+1,1$ & 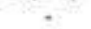 \\
\hline c- & $982 \pm 1,4$ & $\therefore$ & $54,4 \pm 1,3$ & $98,4 \pm 1,1$ \\
\hline
\end{tabular}

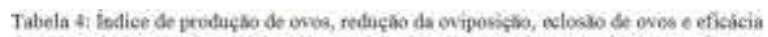

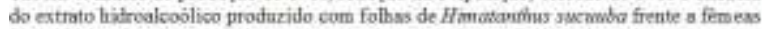

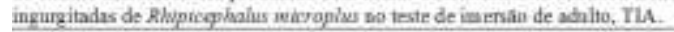

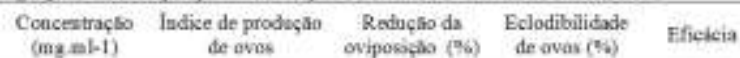

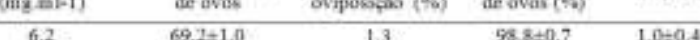

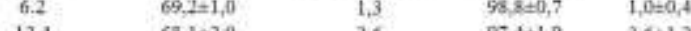
$\begin{array}{lllll}12.4 & 68,1=2,0 & 2,6 & 97,4 \pm 1,9 & 3,6 \pm 1,2 \\ 24.8 & 65,5=2,9 & 6,1 & 95,6 \pm 2,8 & 8,8 \pm 2,1\end{array}$

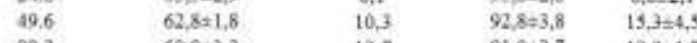

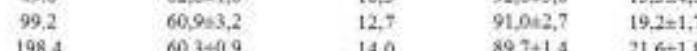

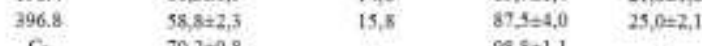
c. $58+1+1,4$ $98,8=1,1$

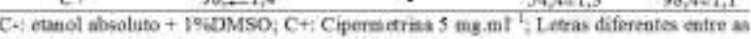
lacunas indican valores estatistieantente difinates (P<0.05)

Interferir nos processos reprodutivos do carrapato é de extrema importância para controlar seu potencial biótico, já que o 
tamanho da progênie é elevada. Alguns trabalhos relataram a eficácia de fitoterápicos em bloquear parcial ou totalmente a ovipostura e/ou eclodibilidade de ovos de fêmeas ingurgitadas (Castro et al.,2014; Ghosh et al., 2015). OS EXTRATOS produzidos no presente estudo demonstrouRAM também está habilidade.

Este é o primeiro trabalho científico abordando 0 efeito acaricida de fitoterápicos produzidos com $H$. suucuba frente a $R$. microplus. Singh et al.(35), utilizaram extratos acetônico, aquoso, clorofórmico, etanólico, hexânico de Murraya koenigii na concentração de 10\% E obtiveram: 81,6; 97,1; 100; 72,1 e $83,3 \%$ de eficácia, respectivamente usando o TIA. Garcia et al. ${ }^{(32)}$ analisaram o efeito acaricida do óleo essencial de Tagetes minuta à 10\%, obtendo eficácia de 92,5 e 79\%, no TPL e TIA, respectivamente. Costa et al. (2008) analisaram a eficácia acaricida dos extratos hidroalcoólicos de citronela, eucalipto e neem à $20 \%$ e observaram efeito de 96; 32 e 17\%, respectivamente, em fêmeas ingurgitadas. Santos et al. (2012) observaram que óleo essencial de Cymbopogon nardus quando utilizado na concentração de 50 e 100\% obteve eficácia de 53 e $73 \%$ no ensaio TIA e 77 e $94 \%$ no ensaio LPT, respectivamente. Olivo et al. (2008) testando diversas concentrações do óleo de citronela $(0,1$; 0,25; 0,5; $1 ; 2 ; 10 ; 25$ e 50) encontraram eficácia sobre fêmeas ingurgitadas de 0,$7 ; 2,8 ; 51,6 ; 79,3 ; 81 ; 87,1 ; 86,7$ e $89,5 \%$, respectivamente. Contudo, vale ressaltar que o uso de extratos em concentrações muito altas podem ser tóxicas para letais, tanto para os parasitas como para os animais. Sendo assim, avaliar a toxicidade dos fitoterápicos é um ponto chave que deve ser abordado em todos os experimentos in vitro.

Os ensaios de toxicidade são elaborados com o objetivo de avaliar os efeitos tóxicos em sistemas biológicos e dimensionar a toxicidade relativa das substâncias para os animais e/ou meio ambiente (PEREIRA et al., 2015). Para tanto o ensaio de letalidade frente ao microcrustáceo $A$. salina Leach. apresenta-se como um método de alta especificidade, barato, seguro e prático (Koutsaftis \& Aoyama, 2007). O teste de toxicidade apresentou $\mathrm{DL}_{50}$ de 1.248 $\mu \mathrm{g} \cdot m L^{-1}$ (1.109 - 1.493; $\left.R^{2}=0,838\right)$. Os resultados encontrados nos testes in vitro aliados aos vistos no teste $\operatorname{com} A$. salina mostram que o extrato produzido possui propriedades antiparasitárias e ao mesmo tempo não é tóxico (DL50>1000 $\mu \mathrm{g} \cdot \mathrm{mL}^{-1}$ ). Somente após a 
comprovação da ausência de efeito deletérios in vitro o fitoterápico poderá ser aprovado para iniciar os testes in vivo (Arcanjo et al., 2012).

O cultivo de plantas medicinais no país ainda é pequeno, todavia encontra-se em ascendência anual, estimulado principalmente pela dificuldade da indústria farmacêutica em obter novos medicamentos sintéticos (Teixeira \& de Melo, 2014). Estes fitoterápicos podem ser usados como alternativa, em locais onde há resistência acaricida múltipla, áreas rurais onde os agricultores não têm acesso aos acaricidas convencionais ou ainda em produções orgânicas de bovinos (Molento et al., 2013). Ressalta-se ainda a tendência de mudança de comportamento dos consumidores, que estão buscando produtos mais saudáveis e com menor uso de produtos químicos durante a produção (Sprenger et al., 2015).

\section{CONCLUSÃO}

Novos estudos devem ser realizados buscando maximizar a eficácia deste extrato, uma vez que foram encontrados resultados promissores.

\section{REFERÊNCIAS}

ABBAS, R.Z.; ZAMAN, M.A.; COLWELL, D.D.; GILLEARD, J.; IQBAL, Z. Acaricide resistance in cattle ticks and approaches to its management: The state of play. Veterinary Parasitology, v. 203, p. 6-20, 2014.

ARCANJO, D.D.R.; ALBUQUERQUE, A.C.M.; MELO-NETO, B.; SANTANA, L.C.L.R.; MEDEIROS, M.; CITÓ, A.M.G.L. Bioactivity evaluation against Artemia salina Leach of medicinal plants used in Brazilian Northeastern folk medicine. Brazilian Journal of Biology, v.72, p.505-509, 2012.

BENNETT G.F. Oviposition of Boophilus microplus (Canestrini) (Acarida: Ixodidae) and Influence of tick size on egg production. Acarologia, v.16, p.5261, 1974.

CARROLL, J.F.; CANTRELL, C.L.; KLUN, J.A.; KRAMER, M. Repellency of two terpenoid compounds isolated from Callicarpa americana (Lamiaceae) against Ixodes scapularis and Amblyomma americanum ticks. Experimental and applied acarology, v. 41, p. 215-224, 2007.

CASTILLO D.; AREVALO J.; HERRERA F.; RUIZ C.; ROJAS R.; RENGIFO E.; VAISBERG A.; LOCK O.; LEMESRE J.L.; GORNITZKA H.; SAUVAIN M. Spirolactone iridoids might be responsible for the Peruvian traditional remedy made with Himatanthus sucuuba (Apocynaceae). Journal of Ethnopharmacol, v.112, p.410- 414, 2007.

CASTRO K.D.C.; LIMA D.F.; VASCONCELOS L.C.; LEITE J.R.S.A.; SANTOS R.C.; NETO A.P.; COSTAJÚNIOR L.M. Acaricide activity in vitro of Acmella oleracea against Rhipicephalus microplus. Parasitology Research, v.113, p.3697-3701. 2014.

CHAGAS, A.D.S. Metodologias in vitro para avaliação de fitoterápicos sobre 
parasitas e resultados de testes a campo. Congresso Brasileiro de Parasitologia Veterinária, p. 13. 2008.

COSTA, F.B.; VASCONCELOS, P.S.S.; Silva, A.M.M.; BRANDÃO, V. M.; Silva, I.A.; TEIXEIRA, W.C.; RITA DE MARIA S.N.; Santos, A.C.G. Eficácia de fitoterápicos em fêmeas ingurgitadas de Boophilus microplus, provenientes da mesorregião oeste do Maranhão, Brasil. Revista Brasileira de Parasitologia Veterinária, v.17, p.83-86, 2008.

DRUMMOND R.O.; ERNST S.E.; TREVINO J.L.; GLADNEY W.J.; GRAHAM O.H. Boophilus annulatus and $B$. microplus: laboratory tests of insecticides. Journal of Economic Entomology, v. 66, p.130-133, 1973.

FERNÁNDEZ-SALAS A.; ALONSODÍAZ M.A.; ACOSTA-RODRÍGUEZ R.; TORRES-ACOSTA J.F.J.; SANDOVALCASTRO C.A.; RODRIGUEZ-VIVAS R.I. In vitro acaricidal effect of tannin-rich plants against the cattle tick Rhipicephalus (Boophilus) microplus (Acari: Ixodidae). Veterinary Parasitology, v. 175, p. 113-118, 2011.

GARCIA, M.V.; MATIAS, J.; BARROS, J.C.; LIMA, D.P.D.; LOPES, R.D. S.; ANDREOTTI, R. Chemical identification of Tagetes minuta Linnaeus (Asteraceae) essential oil and its acaricidal effect on ticks. Revista Brasileira de Parasitologia Veterinária, v. 21, p. 405-411, 2012.

GHOSH S.; TIWARI S.S.; KUMAR B.; SRIVASTAVA S.; SHARMA A.K.; KUMAR S.; RAWAT A.K.S. Identification of potential plant extracts for anti-tick activity against acaricide resistant cattle ticks, Rhipicephalus (Boophilus) microplus

(Acari: Ixodidae). Experimental and Applied Acarology, v.66, p.159-171, 2015.

GRISI, L.; LEITE, R.C.; MARTINS, J.R.S.; BARROS, A.T.M.; ANDREOTTI, R.; CANÇADO, P.H.D.; LEÓN, A.A.P.; PEREIRA, J.B.; VILLELA, H.S.
Reassessment of the potential economic impact of cattle parasites in Brazil. Revista Brasileira de Parasitologia Veterinária, v. 23, n. 2, p. 150-156, 2014

JORDAN, R.A.; SCHULZE, T.L.; DOLAN, M.C. Efficacy of plant-derived and synthetic compounds on clothing as repellents against Ixodes scapularis and Amblyomma americanum (Acari: Ixodidae). Journal of medical entomology, v.49, p.101-106. 2012.

JULIET S.; RAVINDRAN R.; RAMANKUTTY S.A.; GOPALAN A.K.K.; NAIR S.N.; KAVILLIMAKKIL A.K.; GHOSH S. Jatropha curcas (Linn) leaf extract-a possible alternative for population control of Rhipicephalus (Boophilus) annulatus. Asian Pacific Journal of Tropical Disease, v. 2, p. 225229, 2012.

KOUTSAFTIS, A.; AOYAMA, I. Toxicity of four antifouling biocides and their mixtures on the brine shrimp Artemia salina. Science of the Total Environment, v.387, p.166- 174, 2007.

LEITE R.C. Boophilus microplus (Canestrini,1887): susceptibilidade, uso atual e retrospectivo de carrapaticidas em propriedades das regiões fisiográficas da baixada do Grande Rio e Rio de Janeiro: Uma abordagem epidemiológica. Tese (Doutorado em Parasitologia Veterinaria) - Universidade Federal Rural do Rio de Janeiro, Itaguaí. p. 122, 1988.

LUZ, H.S.; SANTOS A.C.G.; MACHADO K.R.G. Prospecção fitoquímica de Himatanthus drasticus Plumel (Apocynaceae), da mesorregião leste maranhense. Rev. bras. plantas medicinais, v.16, p.657-662, 2014.

MADZIMURE J.; NYAHANGARE E.T.; HAMUDIKUWANDA H.; HOVE T.; STEVENSON P.C.; BELMAIN S.R.; MVUMI B.M.; Acaricidal efficacy against cattle ticks and acute oral toxicity of Lippia javanica (Burm F.). Springtrap 
and Tropical Animal Health Production, v.43, p.481-489, 2011.

MOLAN A.; DUNCAN A.; BARRY T.; McNABB W.C. Effects of condensed tannins and crude sesquiterpene lactones extracted from chicory on the motility of larvae of deer lungworm and gastrointestinal nematodes. Parasitology International, v. 52, p. 209-218, 2003.

MOLENTO, M.B.; FORTES, F.S.; BUZATTI, A.; KL SPRENGER, L.K.; COIMBRA, E.; SOARES, L.D. Partial selective treatment of Rhipicephalus microplus and breed resistance variation in beef cows in Rio Grande do Sul, Brazil. Veterinary Parasitology, v. 192, n. 1-3, p. 234-239, 2013.

OLIVO, C.J.; CARVALHO, N.M.; SILVA, J.H.S.; VOGEL, F.F.; MASSARIOL, P.; MEINERZ, G.; AGNOLIN, C.; MOREL, A.F.; VIAU, L.V. Óleo de citronela no controle do carrapato de bovinos. Ciência Rural, Santa Maria, v. 38, p. 406-410, 2008.

POSSIMOSER, D. Et al. Levantamento fitossociológico de espécies nativas com potencial produtivo de fitoterápicos na zona da mata rondoniense. Revista Brasileira de Ciências da Amazônia, v. 1, p.136-140, 2103.

RODRIGUES, E.; DUARTE-ALMEIDA, J.M.; PIRES, J.M. Perfil farmacológico e fitoquímico de plantas indicadas pelos caboclos do Parque Nacional do Jaú (AM) como potenciais analgésicas. Parte I. Revista Brasileira de Farmacognosia, v. 20, p. 981-991, 2010.

ROULSTON W.J.; STONE B.R.; WILSON J.T.; WHITE L.I.; Chemical control of an organophosphorus and carbamate resistant strain Boophilus microplus (Canestrini, 1887). Bull of Entomological Research, v. 58, p. 379391, 1968.

SANTOS, F.C.C.; VOGEL, F.S.F.; MONTEIRO, S.G. Efeito de diferentes concentrações de óleo e tintura de citronela sobre os parâmetros reprodutivos de teleóginas de Rhipicephalus (Boophilus) microplus. Semana: Ciências Agrárias, v.33, p.1141-1148, 2012.

SILVA J.R.A.; PINTO REZENDE C.M.; PINTO A.C.; PINHEIRO M.L.B.; CORDEIRO M.C.; TAMBORINI E.; YOUNG C.M.; BOLZANI V.S. Ésteres triterpênicos de Himatanthus sucuuba (Spruce) Woodson. Química Nova, v.21, p.702-704, 1998b.

SOUZA M.S.; CORDEIRO M.S.; ROSAS E.C.; HENRIQUES M.G.O.M.; SAINI A.C. Inhibition of nitric oxide and interferon-production by iridoids and triterpenes from the roots of Himatanthus sucuuba. Phcog Mag, v. 2, p.216-219, 2006.

SPRENGER, L.K.; BUZATTI, A.; CAMPESTRINI, L.H.; YAMASSAKI, F.T.; MAURER, J.B.B.; BAGGIO, S.F.Z.; MAGALHÃES, P.M.; MOLENTO, M.B. Ovicidal and larvicidal activity of Artemisia annua hydroalcoholic extract against gastrointestinal nematode. Arquivo Brasileiro de Medicina veterinária e Zootecnia, v.67, p.25-31, 2015.

TEIXEIRA, S.A.; DE MELO, J.I.M. Plantas medicinais utilizadas no município de Jupi, Pernambuco, Brasil. IHERINGIA Série Botânica, v. 61, p. 511, 2014.

VILLEGAS, L.F. et al. Evaluation of the wound-healing activity of selected traditional medicinal plants from Peru. Journal of ethnopharmacology, v. 55, p.193-200, 1997.

WOOD C.A.; LEE K.; VAISBERG A.J.; KINGSTON D.G.I.; NETO C.C.; Hammond G.B. A bioactive spirolactone iridoid and triterpenoids from Himatanthus sucuuba. ChemPharm Bull, v.49, p.1477-147, 2011. 